\title{
CSHP Summer Educational Sessions (SES) 2011: Poster Abstracts / Séances éducatives d'été (SÉÉ) 2011 de la SCPH : Résumés des affiches
}

\section{Sunday, August 7, $2011 \bullet$ \\ Dimanche 7 août 2011}

1. Development, Implementation and Assessment of a PharmacistInitiated, Community-Based Immunization Program on Routine Vaccination Rates

2. Feasibility of Assisted Warfarin Dosing (FAST-WARD) by Clinical Pharmacy Support Technicians: An Initial Pilot Project

3. Vomiting of Oral Medications in Pediatric Patients: A Survey on Medication Re-dosing Practices

4. Evaluation of an Electronic Discharge Medication Reconciliation Program during Pilot Phase of Implementation in a Community Hospital Setting

5. Pharmaceutical Care and Patient Satisfaction at a New Atrial Fibrillation Clinic in Vancouver

6. Effect of Drug Formulary Misalignment between Hospital and Provincial Pharmacare on Discharge Medication Discrepancies PPITS (Proton Pump Inhibitor Therapeutic Substitution Study)

7. Institutional Pharmacists' Involvement in and Attitudes towards Research

\section{Monday, August $82011 \bullet$ \\ Lundi 8 août 2011}

1. Economic Evaluation of Dexmedetomidine for Sedation in the Intensive Care Unit

2. Analysis of the Protocols for Opioid Withdrawal as a Harm Reduction Strategy in Hospitalized Patients

3. Follow up Quality Improvement Evaluation of a Pharmacist Managed Warfarin Dosing Service for Outpatient Venous Thromboembolism

4. The Involvement of a Pharmacy Co-op Student in Patient Care Research - A Symbiosis Worth Exploring

5. Management of Acute Seizure at Surrey Memorial Hospital

6. Implementing a Transitional Care Pharmacist Role for Patients at Hospital Discharge

Poster abstracts are published exactly as submitted by the authors and have not undergone any copyediting by the Canadian Journal of Hospital Pharmacy. Le Journal canadien de la pharmacie hospitalière n’a pas soumis les résumés des affiches à une révision linguistique et les publie ici tels que remis par les auteurs.

Development, Implementation and Assessment of a Pharmacist-Initiated, Community-Based Immunization Program on Routine Vaccination Rates

Lindsay Creamer, Andrew Brillant, Jaclyn LeBlanc Horizon Health Network, Saint John, NB

Immunizations are a proven safe and effective preventive health intervention. Despite this, many adults remain deficient in their immunizations. This immunization gap provides an opportunity for health care professionals to enhance people's preventive health care.

The study's objective was to develop, implement, and assess a pharmacist-initiated vaccination program against pneumococcus, tetanus, diphtheria, measles, mumps, and rubella for adults $>18$ years of age attending influenza clinics within the community.
Participants were assessed by a pharmacist for additional New Brunswick publicly-funded adult routine immunizations. Identified vaccinations were recommended to the participant and administered. Of the 47 participants, five (10.6\%) were candidates for measles, mumps \& rubella vaccination, and all five received the vaccine $(100 \%$ acceptance). Thirty-nine participants (83\%) were candidates for tetanus diphtheria vaccination, and $34(72.3 \%)$ received the vaccine (87.2\% acceptance). Thirty-one participants $(66 \%)$ were candidates for pneumococcal vaccination, and $26(55.3 \%)$ received the vaccine (83.9\% acceptance). Most participants (43/47; 91.5\%) were candidates for and received one or more of the three additional immunizations (39/43; 90.7\% acceptance).

A pharmacist-initiated vaccination program demonstrated a clinically significant increase in immunization rates of routine immunizations in adults attending influenza vaccination clinics. 


\section{Feasibility of Assisted Warfarin Dosing (FAST-WARD) by Clinical Pharmacy Support Technicians: An Initial Pilot Project

\author{
Alan Wai, Damen Man, Vincent Mabasa \\ Burnaby Hospital, Burnaby, BC
}

Warfarin dosing by pharmacists is common practice in institutional settings. In this study, we assessed the feasibility of Clinical Pharmacy Support Technicians (CPST) assisting pharmacists in dosing and monitoring hospitalized patients who are prescribed warfarin for atrial fibrillation and/or treatment of thromboembolic events.

CPST received 4 hours of didactic training and 2 weeks of experiential learning using real patient examples. A dosing and monitoring algorithm was developed to guide CPST in recommending an appropriate regimen. CPST collected pertinent data which includes indication, target and present INR, hematology, concurrent medications and potential interactions, and adverse effects; then concisely reported the information to the clinical pharmacists.

In this study, CPST were assessed in their accuracy of data collection, regimen compared to the pharmacist (which pharmacists decided on prior to CPST's report) and the time spent performing this service.

In 30 consecutive patients, results show that CPST are 100\% accurate in data collection, recommended regimen were comparable to clinical pharmacists and completed each recommendation in an average of 12 minutes.

This study demonstrates that when given appropriate education and training, CPST can accurately and efficiently assist clinical pharmacists in dosing and monitoring patients who receive warfarin therapy; and that more investigation is warranted to compare between warfarin dosing by pharmacists and assisted warfarin dosing by CPST.

\section{Vomiting of Oral Medications in Pediatric Patients: A Survey on Medication Re-dosing Practices \\ Jennifer G Kendrick', Kelly Ma'2, Pia DeZorzi ${ }^{1}$, Don Hamilton ${ }^{l}$ ${ }^{I}$ Children's and Women's Health Centre of $B C$, Vancouver, $B C$ ${ }^{2}$ University of British Columbia, Vancouver, $B C$}

Rationale: No policies are in place at our pediatric tertiary care hospital $(\mathrm{C} \& \mathrm{~W})$ to direct clinicians in the event that a child vomits after ingestion of an oral medication. Limited information is available in the literature.

Objectives: The objectives were to characterize the problem of vomiting following administration of an oral medication; to identify the current practice of medication re-dosing; and to collect relevant guidelines and opinions from other pediatric hospitals.

Study Design and Methods: We performed two surveys to identify the current practices and opinions of nurses, pharmacists and physicians who work at pediatric hospitals.

Results: Respondents to the first survey at C\&W reported they encountered the problem of vomiting after administration of oral medications on a weekly $(38.5 \%)$ or monthly $(36.9 \%)$ basis. Most respondents said they followed a general rule to re-dose if vomiting occurs within 30 minutes $(60 \%)$ or 15 minutes $(32.3 \%)$. When asked to rate the importance of eight different factors affecting their clinical decision to re-dose, more than half of respondents indicated time after dose ingestion, medication type, patient status, and visibility of medication in emesis as very important. Sixteen respondents (30.2\%) to the second survey indicated that their pediatric hospital has a guideline regarding what to do in the event that vomiting occurs after ingestion of oral medications. Of these guidelines, most were ward or service specific (66.7\%). Most of respondents stated that their guideline accounts for the time after dose ingestion.

Conclusion: In deciding whether to re-administer a dose, the risk of therapeutic failure must be weighed against the risk of toxicity. This decision likely needs to be individualized for the hospitalized pediatric patient; however, it may be helpful to have a decision-making algorithm or a list of factors to consider for the clinician who encounters this situation.

\section{Evaluation of an Electronic Discharge Medication Reconciliation Program during Pilot Phase of Implementation in a Community Hospital Setting \\ Stacey D'Angelol, ${ }^{1,2}$ Keith Miller, ${ }^{1,2}$,Lucy Feng ${ }^{1,2}$, Jill Parkinsonl, \\ Doug Mitchell \\ 'Pharmacy, Guelph General Hospital, Guelph, ON \\ ${ }^{2}$ School of Pharmacy, University of Waterloo, Kitchener, ON}

Rationale for Report: Discharge medication reconciliation (DMR) is an important service that may increase patient safety. Implementation of an electronic DMR may increase efficiency, allowing pharmacies to provide this service to a greater number of patients.

Description of Concept, Service, Role, or Situation: A longitudinal review of data was completed for patients who received the DMR service from January 12 until April 26, 2011. DMR was provided by a pharmacy co-op student to patients of hospitalist physicians on medical floors at a community hospital.

Steps Taken to Identify and Resolve Problem, Implement Change, or Develop and Implement New Program: Prior to creating the electronic DMR, the discharge prescription was populated manually into a Word processing template. Pharmacy and information management (IM) worked together to create a new documentation process in the electronic software system used by the hospital, Meditech. A list of medications was automatically populated including those from a patient's pharmacy profile (medications prescribed in hospital) and their best possible medication history (BPMH) (medications the patient was on prior to admission). The pharmacy student would reconcile each medication to generate a discharge prescription for the most responsible physician.

Evaluation of Project: An evaluation was completed comparing the manual and electronic DMR. The average number of patients who received a DMR per day increased overall with the electronic process (3.90 vs. $3.08 ; 1.266)$. The percentage of patients discharged from medical floors who received a DMR increased $(43.82 \%$ vs. $38.22 \%$; 1.146). The average total time to complete the service, including reconciliation, updating $\mathrm{BPMH}$, counselling, etc. decreased overall (37.03 minutes vs. 40.73 minutes; 0.909). Likewise, average time to reconcile decreased (19.62 minutes vs. 29.71 minutes; 0.660 ). The average time to update a $\mathrm{BPMH}$ if required dramatically increased (29.79 minutes vs. 13.28 minutes; 2.243).

The Concept's Importance and Usefulness to Current and/or Future Practice: Developing an electronic DMR program increases overall efficiency. The $\mathrm{BPMH}$ has a major influence on time to complete DMR; increasing the number of complete and accurate $\mathrm{BPMHs}$ will likely further increase efficiency of the DMR process. 


\section{Pharmaceutical Care and Patient Satisfaction at a New Atrial Fibrillation Clinic in Vancouver}

Wynnie Lau, Stephen Shalansky, Sonia Basi, Anca Jelescu-Bodos Providence Healthcare, Vancouver, BC

Rationale: An outpatient atrial fibrillation clinic opened at St. Paul's hospital in 2009 and is staffed by a registered nurse, nurse practitioner, pharmacist and four electrophysiologists. With four other clinics recently opened or planned in BC, it was felt that a chart review and patient survey could provide useful information for planning and comparison.

Description of Clinic Care: At clinic entry, patients attend a group education session run by the nurse and pharmacist. A clinical consultation is then scheduled where each patient sees all members of the team. For patients with complex medication histories, the pharmacist and electrophysiologist collaborate on medication treatment decisions. Pharmacists counsel patients who have any changes to their medication regimens, then follow-up by phone to monitor efficacy and adverse effects. Pharmacists also order INR tests and adjust warfarin doses accordingly. Patients who undergo radiofrequency ablation have a narrow target therapeutic range for warfarin on the day of procedure (INR $1.8-2.5$ ), which is a unique challenge.

Evaluation: One hundred patients from first nine months of operation were randomly selected. Mean age was 62.2 years, $62 \%$ male and median CCS SAF score was two. Twenty patients were started on rate control agents and 48 on antiarrhythmics (most commonly dronedarone). Dose adjustments were made for 32 medications while 41 were discontinued. Of the 49 warfarin patients monitored by the pharmacist, the mean time in the therapeutic range was $61 \%$ which compares favourably with reports from other clinics. One of 54 ablation patients had their procedure delayed due to supratherapeutic INR (INR=5.4). Survey responses from 58 patients demonstrated a median satisfaction scores of $19 / 20$ and $86 \%$ indicated that their symptoms were improved or unchanged.

Importance of Concept: Pharmacists at the clinic are required to make complex treatment decisions and assume a high level of independent authority.

\section{Effect of Drug Formulary Misalignment Between Hospital and Provincial Pharmacare on Discharge Medication Discrepancies - PPITS (Proton Pump Inhibitor Therapeutic Substitution Study)}

\author{
Eric Chu, Angela Lo, Fruzina Pataky \\ Vancouver Coastal Health \& Providence Health Care, Vancouver, BC \\ Doson Chua, Linda Tang, Melissa Lo, Ajay Bains \\ St. Paul's Hospital, Vancouver, BC
}

Rationale: Medication discrepancies can occur on hospital admission, transfer and discharge. Therapeutic substitution within a drug class for in-hospital medication orders is a common practice. Proton pump inhibitors (PPI) is one drug class that is commonly therapeutically substituted for all PPI orders prescribed during hospitalization. However, the formulary PPI in hospital may not align with the provincial formulary PPI for non-hospitalized patients. This discordance between the provincial and hospital formulary may lead to discharge discrepancies. Objectives: To evaluate the effect the misalignment of formulary PPI between hospital and provincial Pharmacare on medication discharge discrepancies.

Study Methods: A retrospective chart from 2006-2010 of all patients admitted to the St. Paul's Hospital (SPH) cardiology units that was prescribed a PPI during hospitalization. The inclusion criteria were patients that were prescribed a PPI prior to admission and on discharge from hospital. Patients who expired, transferred to another ward, had an intolerance to particular PPI, had no medical or medication history available and had no record of their discharge prescription (written on a SPH triplicate copy prescription form) were excluded. A medication discharge discrepancy was defined as being discharged home on a PPI that was different than the PPI prior to hospitalization.

Results: 2,408 patients were screened and 253 patients were included in the study. Rabeprazole was the formulary PPI for the British Columbia (BC) Provincial Pharmacare program throughout the study period. Rabeprazole was the SPH formulary PPI during 2006-2008 (Period A, n=88). Lansoprazole and esomeprazole was the SPH formulary PPI during 2008-2010 (Period B, $n=165$ ). The incidence of discharge medication discrepancies was significantly higher when the hospital and provincial formulary PPI did not match (Period B = 49\%) compared when the hospital and provincial formulary PPI were aligned (Period $\mathrm{A}=27 \%$ ). This misalignment of formulary PPI was associated with a $22 \%$ absolute risk increased of a medication discharge discrepancy ( $\mathrm{p}=0.0008)$.

Conclusion: Misalignment of formulary PPI between hospital and provincial Pharmacare was associated with a significant increase in discharge medication discrepancies.

\section{Institutional Pharmacists' Involvement in and Attitudes towards Research}

S L Koshman', J Blais', S Walter', R T Tsuyuki', E Manchuk², D Eurich ${ }^{3}$

${ }^{1}$ Faculty of Medicine, University of Alberta, Edmonton, $A B$

${ }^{2}$ Pharmacy Services, Alberta Health Services, Edmonton, $A B$

${ }^{3}$ School of Public Health, University of Alberta, Edmonton, $A B$

Rationale: Research is critical for the future of pharmacy. Institutional practice requires a specialized body of knowledge that supports practice patterns and processes. Little is known about institutional pharmacists' (IP) involvement in research.

Objectives: To describe IP involvement in research activities and identify enablers and barriers of research involvement, as well as attitudes and perceptions surrounding research.

Methods: A cross-sectional internet survey of all IP within Alberta Health Services, Alberta. Survey development utilized adaptation of existing surveys in the literature, expert opinion, and pre-testing with a group of IP. The survey, using Survey MonkeyTM, was distributed via a master list though Pharmacy Services administration. In addition to the initial invitation, 2 follow-up emails were sent to encourage participation. Internal reliability of the survey was determined by using Cronbach's alpha. Data analysis was descriptive.

Results: Overall response rate was 45\% (286/636). Respondents were mostly female $(79 \%)$, practiced in a tertiary care centre (70\%) and were employed full-time (70\%). Years of institutional practice were equally distributed. Sixty-percent $(n=168)$ responded that they had been involved in research. Of those involved in research, the most frequently reported research activities were dispensing study medications $(57 \%)$, identifying or enrolling patients $(55 \%)$, and reviewing research reports $(54 \%)$. The most common types of research included clinical $(58 \%)$ and health services research (28\%). Almost all respondents (99\%) felt that pharmacists should be involved in research. Pharmacists generally agreed that research was important to the profession of pharmacy, but that they required more time and training to become more involved in research activities.

Conclusion: While the majority of institutional pharmacists in Alberta have had involvement in research projects, few were involved in 
leading research. There is strong support from pharmacist to be involved in research; however, most feel that they lack the skills and support to do these activities.

\section{Economic Evaluation of Dexmedetomidine for Sedation in the Intensive Care Unit}

$J$ Lachaine $^{l}$, C Beauchemin ${ }^{\prime}$, R Amer $^{2}$

${ }^{I}$ Faculty of Pharmacy, University of Montreal, Montréal, QC

${ }^{2}$ Hospira Healthcare Corporation, Montréal, QC

Objective: Dexmedetomidine is an alpha- 2 receptor agonist used in continuous infusion for the sedation of critically ill patients in intensive care unit who are intubated and mechanically ventilated. Compared to midazolam in the sedation of intensive care unit patients, dexmedetomidine showed a decrease in time spent on ventilator, fewer episodes of delirium and reduced incidence of tachycardia and hypertension. The aim of this study was to assess the economic impact, in a Canadian context, of dexmedetomidine for sedation in intensive unit care compare with midazolam, a GABA agonist.

Methods: This economic evaluation was performed using a costconsequences analysis, according the perspective of Canadian Health Care system. The time horizon chosen is an intensive care unit stay with a maximum length of 30 days. Clinical data were obtained from a prospective randomized, double-blinded trial by Riker and al. comparing dexmedetomidine and midazolam. Costs considered in this evaluation were those of the medications, of the mechanical ventilation, of the delirium episodes, and those associated with adverse events requiring an intervention. All costs were adjusted to 2010 and were reported in Canadian dollars.

Results: The average cost of medication was higher with dexmedetomidine $(\$ 1,930)$ than with midazolam $(\$ 180)$, but the average cost associated with mechanical ventilation and with the management of delirium were lower with dexmedetomidine $(\$ 2,939$ and $\$ 3,630$ respectively) than with midazolam $(\$ 4,448$ and $\$ 5,149)$. Overall cost per patient with dexmedetomidine $(\$ 8,525)$ was lower than with midazolam $(\$ 9,817)$. Deterministic sensitivity analysis confirmed the robustness of this difference.

Conclusion: The results of this cost-consequences analysis indicated that the use of dexmedetomidine is a favorable strategy in terms of clinical consequences and economic impact compare to midazolam. Compared to midazolam, dexmedetomidine is a less expensive strategy associated with a lower occurrence of delirium and a shorter duration of mechanical ventilation.

\section{Analysis of the Protocols for Opioid Withdrawal as a Harm Reduction Strategy in Hospitalized Patients \\ Andrew Brillant \\ Horizon Health Network, Saint John, NB}

Rationale: The Protocols for Opioid Withdrawal (POW) are a harm reduction strategy to manage opioid addictions while patients are hospitalized for medical reasons. POW incorporates narcotic substitution, collection of a drug-use inventory, health screening, and immunizations. Preventing withdrawal symptoms may lead to more people completing their medical treatment plan; meanwhile the health screening and immunizations aim to prevent future illness.

Objectives: To determine how POW interventions are utilized for inpatients initiated on methadone, including the frequency of discharges against medical advice (AMA) and plans to continue an outpatient methadone maintenance program.
Methodology: A retrospective review was performed on patient charts with an order for diluted methadone from January 2008 to December 2009. Patients were excluded if they were prescribed methadone as a tablet, as undiluted liquid, or as continuation of therapy.

Results: Twenty-three patients were analysed using descriptive statistics and Pearson correlations. No patient had all components of POW implemented (drug inventory, signed patient agreement, completion of relevant laboratory investigations, updated immunizations, and use of the Clinical Opioid Withdrawal Scale [COWS]). The COWS was the most commonly used component $(73.9 \%)$ while immunizations were least likely to be completed (8.7\%). Typically methadone was prescribed on the first day of hospitalization at a mean dosage of $35 \mathrm{mg}$ daily (range $15-70 \mathrm{mg}$ ). There were 17 patients (73.9\%) prescribed an additional $5 \mathrm{mg}$ methadone dose if a COWS score was $>6$. Discharges AMA were reported for $3(13 \%)$ of the patients. Outpatient methadone maintenance was planned for 21 patients $(91.3 \%)$.

Conclusion: More could be done to improve the health screening and immunization of inpatients with opioid addiction. Initiating inpatients on methadone may improve the chance of continuing methadone maintenance therapy in the community; however, it appears that opioid withdrawal is not the main factor for discharges against medical advice.

\section{Follow up Quality Improvement Evaluation of a Pharmacist Managed Warfarin Dosing Service for Outpatient Venous Thromboembolism}

Patrick Fitch, Julie Mistri

Winnipeg Regional Health Authority Pharmacy Program, Winnipeg, MB Adrian Gulowaty

Faculty of Pharmacy, University of Manitoba, Winnipeg, $M B$

Rationale: In April 2008, we implemented a pharmacist managed warfarin dosing service for outpatients initiated on warfarin for treatment of venous thromboembolism (VTE), using one of two previously validated dosing nomograms (initial dose $5 \mathrm{mg}$ or $10 \mathrm{mg}$ ). In 2009, we performed a quality improvement evaluation to assess the efficacy and safety of the service, and subsequently altered the pharmacy staffing model to allow for greater staffing consistency. The purpose of this project is to evaluate the effectiveness of the modified service.

Description of Project: We conducted a retrospective audit of pharmacy profiles for all patients referred to the service between September 15, 2010 and February 20, 2011. Data was collected to determine appropriate nomogram selection, time to achieve therapeutic INR, bleeding complications and interventions, reasons for deviating from the nomogram and the number of patients ineligible for enrollment in the program.

Evaluation: Eighteen patients were included (mean age $55.2 \mathrm{yrs}, 61 \%$ male). The appropriate nomogram was selected in $72 \%$ of patients. By day 5, 50\% achieved therapeutic INR as compared to 35\% in 2009 . The project also showed that $72 \%$ patients achieved therapeutic INR at the end of both nomograms. Thirty three percent of patients experienced a supratherapeutic INR value $>3.0$. The project revealed $28 \%$ patients deviated from the nomograms compared to $66.7 \%$ in 2009. The majority (90\%) of patients presenting with VTE were eligible for enrollment in the pharmacist managed warfarin dosing service.

Conclusion: The implemented recommendations of the 2009 study have lead to an improvement in the service by increasing the proportion of patients reaching therapeutic level at day 5 and decreasing the number of nomogram deviations. The pharmacist managed warfarin dosing program continues to be a safe and effective method of initiating warfarin therapy in outpatients requiring anticoagulation. 


\section{The Involvement of a Pharmacy Co-op Student in Patient Care Research - A Symbiosis Worth Exploring}

Melanie MacInnis, Hamilton Health Sciences, Hamilton, ON Marc-André Gravel, School of Pharmacy, University of Waterloo, Kitchener, $O N$

Rationale: Many hospital pharmacists are struggling to find the time, resources, and funding to allow them to pursue quality improvement projects (QIP). Certain aspects of QIP, such as data collection, are often not regarded as the best use of a pharmacist's time. To address this problem, our institution hired a pharmacy co-op student to conduct data collection pertaining to medication safety in the perioperative services program.

Description of Concept: The goal of the project was to identify how and where medication discrepancies occur when patients are scheduled for elective surgeries, as well as to evaluate the impact of having a dedicated pharmacy team member recording patients' best possible medication histories (BPMH) in a preoperative clinic.

Steps Taken to Develop and Implement New Program: The student had the opportunity to spend a total of 6 weeks recording BPMH with patients in the preoperative clinic. The student also followed patients in both the same-day surgery department and the post-operative wards. By doing so, the student was able to identify areas where medication information transfer could be improved and therefore reduce errors.

Evaluation of Project: The results and recommendations were presented to various stakeholders. The impact the pharmacy student had in the preoperative clinic was such that the Perioperative Services Program has hired a full time pharmacy technician to assist with BPMH in the clinic. Furthermore, the student's recommendations were synthesized and will be integrated in an updated version of the perioperative medication history form.

Importance and Usefulness to Current and/or Future Practice: The impact of the student-led project has resulted in changes that will facilitate patient care, promote pharmacy practice, and enhance medication safety at interfaces of care. It has also fostered valuable patient care experience and introduced project management skills to a pharmacy undergraduate student.

\section{Management of Acute Seizure at Surrey Memorial Hospital \\ T Magnusson, A Alleyne, J Diggle \\ Surrey Memorial Hospital, Surrey, BC}

Background: Over 250 patients admitted to Surrey Memorial Hospital (SMH) each year are treated for acute seizure during their hospital stay. SMH Patient Care Guidelines (1996) recommend that a Code Blue be initiated after a "prolonged seizure", defined as $>60$ seconds in duration. Current recommendations indicate that only supportive measures are to be performed during the first 5 minutes of a seizure; and that pharmacologic treatment for seizure cessation is started if the seizure continues beyond 5 minutes. SMH neurologists report that many patients suffering from acute seizures are administered a benzodiazepine within the first five minutes, without waiting for spontaneous resolution of the seizure. This "use of a drug with no indication" may lead to unnecessary morbidity and mortality, and possibly an increase in length of stay.

Objectives: To investigate and describe the current management of acute seizure at SMH. To investigate the impact on length of stay as a result of using a pharmacological treatment for seizure cessation during the first five minutes of seizure activity without waiting for spontaneous resolution of symptoms.

Methods: Retrospective chart review of adults experiencing an inhospital seizure 01JA09 to 22JL10.

Results:

- A convenience sample of 119 charts were randomly selected from a possible 388 patients

- $77 \%$ of seizures were less than five minutes in length - $43 \%$ of these patients received seizure-abortive therapy

- Lorazepam was the primary pharmacological therapy used to stop seizures

$-88 \%$ of patients were under-dosed using $0.1 \mathrm{mg} / \mathrm{kg}$ for seizure cessation

- There was a trend to increased length of stay associated with use of benzodiazepines ( $\mathrm{p}=0.351$, Mann Whitney Test)

Conclusions: Management of acute seizures is inconsistent and not in compliance with current recommendations. Almost half of short duration seizures are unnecessarily managed with seizure-abortive therapy. Current SMH guidelines for management of acute seizures require revision.

\section{Implementing a Transitional Care Pharmacist Role for Patients at Hospital Discharge}

Emily Charlesworth, Sarah Edwards, Ryan Petryschuk, King Wong, Kori Leblanc, Olavo Fernandes

Toronto General Hospital, University Health Network, Toronto, ON

Rationale: Patients being discharged from hospital are at risk for adverse drug events, medication discrepancies and drug therapy problems. The Transitional Care Pharmacist Pilot Project is a quality initiative to: 1) improve continuity of care for patients at discharge; 2 ) explore out-patient $(\mathrm{OP})$ pharmacy revenue generating opportunities. Description: Based in the OP pharmacy, the Transitional Care Pharmacist partners with in-patient (IP) pharmacists by filling discharge prescriptions, providing medication education and transferring information to the patient's home pharmacy.

Steps Taken: During the initial three months, patients being discharged home from a Vascular Surgery unit were offered the services of the Transitional Care Pharmacist. From this experience, standard procedures, process maps, and fax forms for referral and transfer to home pharmacies were developed. In the following six months, services were expanded to the Cardiovascular Surgery patients.

Evaluation: Benefits of the program are: 1) Enhanced patient services at discharge (medication delivery to IP units, focused medication teaching, and convenience for out-of-town patients); 2) Strengthened IP and OP pharmacist collaboration; 3) Improved relationships with home pharmacies. Each home pharmacy received a medication list with changes and relevant follow-up instructions. Challenges that curtailed recruitment for this program included: 1) Narrow window between prescriptions being finalized and time of discharge; 2) A patient population that was frequently discharged to other institutions; 3) Patient preference for home pharmacy. Overall, 111 patients were enrolled with an average of 6 prescriptions per patient. IP pharmacist time to recruit and receive consent from patients, transfer information and follow-up with the OP pharmacist was 15 minutes on average.

Importance: This quality initiative was developed to improve continuity of care for patients. It has improved communication between the hospital and community pharmacies. We believe a similar model can be implemented across other IP units at our hospital to benefit more patients. 